\title{
Some aspects of tight-binding approach in chemisorption theory
}

\author{
Yu.Rudavskii, G.Ponedilok, Yu.Petriv \\ Lviv Polytechnic State University \\ 12 S.Bandera Str., 79013 Lviv, Ukraine
}

Received August 26, 2000

\begin{abstract}
The quantum-statistic problem of atoms chemisorption with hydrogen-like electronic structure on semiconductor and dielectric crystalline surfaces is considered. The superficial states are described in tight-binding approximation. The self-consistent system of equations for calculating the charge and spin-polarized states of the adsorbed atom in Hartree-Fock approximation is derived.
\end{abstract}

Key words: adatom, chemisorption, surface, chemisorption energy

PACS: $73.20 . H, 82.65 . Y$

\section{Introduction}

The quantum atom chemisorption theory takes an important place in the physics of metal surface, semiconductors and detectors. This problem has been considered in numerous papers (see [1-4] and their references). Based on different theoretical models and methods, essential success in understanding the chemisorption mechanisms and in qualitative explanation of the seen phenomena was achieved. The most frequently used are the method of density functional, molecular orbital methods, clusters models. But still a lot of principal questions of quantum chemisorption theory remain open. In particular, it corresponds to construction both of adequate microscopic models and the development of effective approximate analysis methods of these models condition of the absence of small parameters, that does not permit to employ a standard perturbation theory. From this point of view, the microscopic models, which are built on the ideas of the well-known in quantum solid state theory Anderson model [5], are perspective. Firstly, in such models the physical meaning of parameters is quite obvious, that permits to choose and estimate them for specific systems. Secondly, the Anderson model is well established in many aspects. It permits to transmit the approved results and methods on chemisorption theory problems. The microscopic model of chemisorption, based on the main ideas of Anderson model was proposed in Newns paper [6]. The problem is solved using the HartreeFock approximation: the chemisorption energy and the charge state of chemisorbed 
atom are calculated. The model, suggested initially in [6], was generalized later. In particular, the Coulomb electron-electron interaction was taken into consideration, the effects of non-orthogonality of adatom orbital to atom surface orbital and overfilling of one-site basis were studied. The chemisorption theory studied at papers $[6,7]$, was built on supposition that the substrate is found in paramagnetic state.

In present paper the extended version of microscopic Newns-Anderson [6] model is taken as the base. The space non-homogeneousness of the model parameters, Coulomb and exchange interactions in the "the surface - adatom" system, the local potential-field effects of surface layer ions additionally are taken into consideration. The system of equations for calculating the charge and spin-polarized states of the adsorbed atom and binder energy of adatom with the surface in Hartree-Fock approach are derived. Unlike Newns paper [6], where the chemisorption on the surface of metals was investigated, in our model we take into account the correlation effects, which permit to extend the model to atoms chemisorption problem on dielectric and semiconductor surfaces.

\section{The microscopic model of chemisorption}

A laboratory system of coordinates is competent so that a plane $x O y$ is situated on half-limited crystal surface. In half-space $Z \geqslant 0$ a separate atom is found, on electronic structure of valence envelope of which has hydrogen-like structure. An electronic surface structure of limited environment is described in tight-binding approach. It means that a dominating role in the course of atom chemisorption is played by the electronic states of surface atoms. Let the $\mathbf{R}_{j}=R_{x} \mathbf{e}_{x}+R_{y} \mathbf{e}_{y}, j=\overline{1, N}$ are the coordinates of surface atoms, and $\mathbf{R}_{0}=Z_{0} \mathbf{e}_{z}$ is the adatom position.

A Hamilton operator of "surface-adatom" system in the secondary quantization image has the following form

$$
\hat{H}=C+\hat{H}_{0}+\hat{H}_{\text {corr }} .
$$

The effective one-particle part of operator (1) has the following structure

$$
\begin{aligned}
\hat{H}_{0}= & \sum_{1 \leqslant j \leqslant N} \sum_{\sigma=\uparrow, \downarrow} \mathcal{E}_{j, \sigma} a_{j \sigma}^{+} a_{j \sigma}+\sum_{\sigma=\uparrow, \downarrow} E_{\sigma} d_{0 \sigma}^{+} d_{0 \sigma}+\sum_{1 \leqslant i \neq j \leqslant N} \sum_{\sigma=\uparrow, \downarrow} T_{i j} a_{i \sigma}^{+} a_{j \sigma} \\
& +\sum_{1 \leqslant i \leqslant N} \sum_{\sigma=\uparrow, \downarrow}\left(V_{i, 0} a_{i \sigma}^{+} d_{0 \sigma}+V_{0, i} d_{0 \sigma}^{+} a_{i \sigma}\right) .
\end{aligned}
$$

Here $a_{j \sigma}^{+}\left(a_{j \sigma}\right)$ and $d_{0 \sigma}^{+}\left(d_{0 \sigma}\right)$ are electrons Fermi creation (annihilation) operators on the $\mathbf{R}_{j}$-th surface atom and on the adatom, accordingly, which is described by s-type atom wave functions $\Psi\left(\left|\mathbf{r}-\mathbf{R}_{i}\right|\right)$ and $\varphi\left(\left|\mathbf{r}-\mathbf{R}_{i}\right|\right)$. The respective energy levels are the $\varepsilon_{0}$ and $E_{0}$. Further in the paper we employed the notations $\hat{n}_{j, \sigma}=a_{j \sigma}^{+} a_{j \sigma}$ and $\hat{n}_{0, \sigma}=d_{0 \sigma}^{+} d_{0 \sigma}$ for operators of the number of electrons that localized on atomic states with spin projection $\sigma$ on the axis of quantization. Also, the $\hat{n}_{0}=\hat{n}_{0, \uparrow}+\hat{n}_{0, \downarrow}$ is the operator of the total number of electrons, localized on $s$-orbital of the adsorbed atom and the respective operator for $j$-th surface atom is $\hat{n}_{j}=\hat{n}_{j, \uparrow}+\hat{n}_{j, \downarrow}$. 
The renormalized energy of a localized electron state on the adatom has the following structure

$$
E_{\sigma}=E_{0}+U_{0}\left\langle\hat{n}_{0,-\sigma}\right\rangle+\sum_{j=1}^{N}\left[\Phi_{\mathrm{s}}\left(\left|\mathbf{R}_{0}-\mathbf{R}_{j}\right|\right)+U\left(\left|\mathbf{R}_{0}-\mathbf{R}_{j}\right|\right)\left\langle\hat{n}_{j}\right\rangle\right] .
$$

Here and further in the paper, the symbol $\langle\ldots\rangle$ mean thermodynamically averaged by Gibbs distribution. The second term at the right part of (3) describes Hubbard level splitting due to the intensity of Coulomb interaction $U_{0}$. The latter term characterizes the adatom level shift taking into account the total action of surface atoms. The value $\Phi_{\mathrm{s}}\left(\left|\mathbf{R}_{j}-\mathbf{R}_{0}\right|\right)=\int \varphi^{2}\left(\mathbf{r}-\mathbf{R}_{0}\right) V_{\mathrm{s}}\left(\left|\mathbf{r}-\mathbf{R}_{j}\right|\right) \mathrm{d} \mathbf{r}$ is the effective electron energy in the potential field of $\mathbf{R}_{j}$-th surface atom and $V_{\mathrm{s}}\left(\left|\mathbf{r}-\mathbf{R}_{j}\right|\right)=-Z_{\mathrm{s}} e^{2} /\left|\mathbf{r}-\mathbf{R}_{j}\right|$.

The Coulomb integral

$$
U\left(\left|\mathbf{R}_{0}-\mathbf{R}_{j}\right|\right)=\iint \varphi^{2}\left(\mathbf{r}-\mathbf{R}_{0}\right) W(|\mathbf{r}-\mathbf{r} /|) \Psi^{2}\left(\mathbf{r}^{\prime}-\mathbf{R}_{j}\right) \mathrm{d} \mathbf{r} \mathrm{d} \mathbf{r}^{\prime}
$$

is proportional to the average energy of the electron at the field of effective surface atom charge and for $s$-orbital this energy depends only on the distance between the atoms. From expression (3) we can see that atomic level $E_{0}$ of the adsorbed atom has got a shift under the action of total potential of surface atoms.

The energy of localized electron state on $\mathbf{R}_{j}$-th surface atom is as follows

$$
\mathcal{E}_{j, \sigma}=\mathcal{E}_{0}+U_{\mathrm{s}}\left\langle\hat{n}_{j,-\sigma}\right\rangle+\Phi_{\mathrm{a}}\left(\left|\mathbf{R}_{j}-\mathbf{R}_{0}\right|\right)+U\left(\left|\mathbf{R}_{j}-\mathbf{R}_{0}\right|\right)\left\langle\hat{n}_{0}\right\rangle .
$$

In this expression, the parameter $\Phi_{\mathrm{a}}\left(\left|\mathbf{R}_{j}-\mathbf{R}_{0}\right|\right)=\int \Psi^{2}\left(\mathbf{r}-\mathbf{R}_{j}\right) V_{\mathrm{a}}\left(\left|\mathbf{r}-\mathbf{R}_{0}\right|\right) \mathrm{d} \mathbf{r}$ characterizes level shift of $j$-th surface atom under adatom potential action, and $V_{\mathrm{a}}\left(\left|\mathbf{r}-\mathbf{R}_{0}\right|\right)=-Z_{\mathrm{a}} e^{2} /\left|\mathbf{r}-\mathbf{R}_{0}\right|$. Here $V_{\mathrm{s}}\left(\left|\mathbf{r}_{i}-\mathbf{R}_{j}\right|\right)$ and $V_{\mathrm{a}}\left(\left|\mathbf{r}_{i}-\mathbf{R}_{0}\right|\right)$ are electron potential energy in $\mathbf{R}_{j}$ surface atom and adsorbed atom $\mathbf{R}_{0}$ field, accordingly. The two last terms in the right part of (4) describe the electrons correlation effects, like in expression (3). The parameter $U_{\mathrm{s}}$ is the energy of Hubbard correlation of electrons that are localized on $s$-orbital of the surface atom.

The operator of Coulomb electron-electron correlation in (1) is

$$
\begin{aligned}
\hat{H}_{\mathrm{corr}}= & \frac{U_{0}}{2} \sum_{\sigma=\uparrow, \downarrow} \delta \hat{n}_{0 \sigma} \delta \hat{n}_{0,-\sigma}+\frac{U_{\mathrm{s}}}{2} \sum_{1 \leqslant j \leqslant N} \sum_{\sigma=\uparrow, \downarrow} \delta \hat{n}_{j \sigma} \delta \hat{n}_{j,-\sigma} \\
& +\sum_{1 \leqslant j \leqslant N} U\left(\left|\mathbf{R}_{j}-\mathbf{R}_{0}\right|\right) \delta \hat{n}_{j} \delta \hat{n}_{0} .
\end{aligned}
$$

In this expression we employed notes $\delta \hat{n}_{j \sigma}=\hat{n}_{j \sigma}-\left\langle\hat{n}_{j \sigma}\right\rangle, \delta \hat{n}_{0 \sigma}=\hat{n}_{0 \sigma}-\left\langle\hat{n}_{0 \sigma}\right\rangle$ for the operators of fluctuations of number of electrons localized on the atoms. The values $\left\langle\hat{n}_{j}\right\rangle=\left\langle\hat{n}_{j \uparrow}\right\rangle+\left\langle\hat{n}_{j \downarrow}\right\rangle,\left\langle\hat{n}_{0}\right\rangle=\left\langle\hat{n}_{0 \uparrow}\right\rangle+\left\langle\hat{n}_{0 \downarrow}\right\rangle$ are the thermodynamically averaged number of electrons, localized on $j$-th surface atom and on the chemisorbed atom, accordingly.

Non-operator part of Hamiltonian (1)

$$
C=-U_{0}\left\langle\hat{n}_{0 \uparrow}\right\rangle\left\langle\hat{n}_{0 \downarrow}\right\rangle-\sum_{1 \leqslant j \leqslant N} U_{\mathrm{s}}\left\langle\hat{n}_{j \uparrow}\right\rangle\left\langle\hat{n}_{j \downarrow}\right\rangle-\sum_{1 \leqslant j \leqslant N} \Phi\left(\left|\mathbf{R}_{j}-\mathbf{R}_{0}\right|\right)\left\langle\hat{n}_{j}\right\rangle\left\langle\hat{n}_{0}\right\rangle
$$

is the effective electrostatic energy of correlation of electrons. 


\section{Hartree-Fock approach. The case of crystalline surface}

Hartree-Fock approach permits a qualitative study of physics of chemisorption processes. However, in the approach, the microscopic models of chemisorption have been studied by now, if not to take into account directly the quantum-statistical calculation and computer simulation. In Hartree-Fock approach, in the operator (1), the energy of electron-electron correlation $\hat{H}_{\text {corr }}$ is disregarded. The effective Hamilton operator for this approach is as follows:

$$
\hat{H}_{\mathrm{HF}}=C+\hat{H}_{0} \text {. }
$$

In the case of crystalline surface, it is convenient to take transformation directly in momentum space. Then Hamilton operator of the model in Hartree-Fock approach is

$$
\begin{aligned}
\hat{H}_{0}= & \sum_{\mathbf{k} \in B} \sum_{\sigma=\uparrow, \downarrow} t_{\mathbf{k}} a_{\mathbf{k} \sigma}^{+} a_{\mathbf{k} \sigma}+\sum_{\sigma=\uparrow, \downarrow} E_{\sigma} d_{0 \sigma}^{+} d_{0 \sigma}+\sum_{\mathbf{k}, \mathbf{q} \in B} \sum_{\sigma=\uparrow, \downarrow} \Delta_{\mathbf{q}, \sigma} a_{\mathbf{k} \sigma}^{+} a_{\mathbf{k}-\mathbf{q}, \sigma} \\
& +\sum_{\mathbf{k} \in B} \sum_{\sigma=\uparrow, \downarrow}\left(V_{\mathbf{k}} a_{\mathbf{k} \sigma}^{+} d_{0 \sigma}+V_{\mathbf{k}}^{*} d_{0 \sigma}^{+} a_{\mathbf{k} \sigma}\right) .
\end{aligned}
$$

The Fermi operators of annihilation and creation of electrons at $\{\mathbf{k}, \sigma\}$ states are as follows:

$$
a_{\mathbf{k} \sigma}=\frac{1}{\sqrt{N}} \sum_{j=1}^{N} a_{j \sigma} \mathrm{e}^{-\mathrm{i} \mathbf{k} \mathbf{R}_{j}}, \quad a_{\mathbf{k} \sigma}^{+}=\frac{1}{\sqrt{N}} \sum_{j=1}^{N} a_{j \sigma}^{+} \mathrm{e}^{\mathrm{ikR_{j }}} .
$$

For a semi-limited media, as remarked in [6], the wave vector $\mathbf{k}$ consists of parallel to crystal surface components $\mathbf{k}_{\|}$, which acquires uninterrupted values within the first two-dimensional Brillouin zone, and perpendicular to crystal surface components $\mathbf{k}_{z}$, which acquires discrete values. In this section, under wave vector $\mathbf{k}$ we understand only parallel constituent of wave vector, skipping additional symbols in designations. Also, the perpendicular constituent of wave vector acquires only one discrete value is supposed. For physical sense, such a simplification is equivalent to supposition that in chemisorption processes only one zone is important that has lowermost energy of superficial states.

Matrix elements of electron transfers between localized surface atom states are as follows:

$$
T_{i j}=\frac{1}{N} \sum_{\mathbf{k}} t_{\mathbf{k}} \mathrm{e}^{\mathrm{i} \mathbf{k}\left(\mathbf{R}_{i}-\mathbf{R}_{j}\right)}, \quad t_{\mathbf{k}}=\sum_{j=1}^{N} T_{i j} \mathrm{e}^{-\mathrm{i} \mathbf{k}\left(\mathbf{R}_{i}-\mathbf{R}_{j}\right)} .
$$

The value $t_{\mathbf{k}}$ is the electrons spectrum of surface Hubbard subbands. The Fouriercoefficients of pseudopotential of electrons dispersion on non-homogeneous surface are as follows:

$$
\Delta_{\mathbf{p}, \sigma}=\frac{1}{N} \sum_{1 \leqslant j \leqslant N} \Delta_{j \sigma} \mathrm{e}^{-\mathrm{ipR_{j }} .}
$$

The coefficient $\Delta_{j \sigma}$ describes heterogeneous level shift of $j$-th surface atom under the action of correlation effects of Coulomb type

$$
\Delta_{j, \sigma}=U_{\mathrm{s}}\left\langle\hat{n}_{j,-\sigma}\right\rangle+\Phi_{\mathrm{a}}\left(\left|\mathbf{R}_{j}-\mathbf{R}_{0}\right|\right)+U\left(\left|\mathbf{R}_{j}-\mathbf{R}_{0}\right|\right)\left\langle\hat{n}_{0}\right\rangle .
$$


Fourier-components of matrix potential elements, which characterize the hybridization processes of localized surface electrons and the electrons localized on the adatom are as follows:

$$
V_{\mathbf{k}}=\frac{1}{\sqrt{N}} \sum_{j=1}^{N} V_{j, 0} \mathrm{e}^{-\mathrm{ikR_{j }}} .
$$

For Hamilton operator $\hat{H}=C+\hat{H}_{0}$ the following matrix has been calculated.

$$
\mathbf{G}^{\sigma}(\omega)=\left(\begin{array}{cc}
\left\langle\left\langle a_{\mathbf{k} \sigma} \mid a_{\mathbf{q} \sigma}^{+}\right\rangle\right\rangle_{\omega} & \left\langle\left\langle a_{\mathbf{k} \sigma} \mid d_{0 \sigma}^{+}\right\rangle\right\rangle_{\omega} \\
\left\langle\left\langle d_{0 \sigma} \mid a_{\mathbf{q} \sigma}^{+}\right\rangle\right\rangle_{\omega} & \left\langle\left\langle d_{0 \sigma} \mid d_{0 \sigma}^{+}\right\rangle\right\rangle_{\omega}
\end{array}\right),
$$

elements of which are frequency images of two-time anticommutative one-electron Green functions $\left\langle\left\langle\hat{A}(t) \mid \hat{B}\left(t_{0}\right)\right\rangle\right\rangle=-\mathrm{i} \Theta\left(t-t_{0}\right)\left\langle\left[\hat{A}(t), \hat{B}\left(t_{0}\right)\right]_{+}\right\rangle$.

Equation of motion for Green functions matrix elements (13) are as follows:

$$
\begin{aligned}
& \left(\omega-t_{\mathbf{k}}\right)\left\langle\left\langle a_{\mathbf{k} \sigma} \mid a_{\mathbf{k}^{\prime} \sigma}^{+}\right\rangle\right\rangle_{\omega}=\frac{1}{2 \pi} \delta_{\mathbf{k}, \mathbf{k}^{\prime}}+V_{\mathbf{k}}\left\langle\left\langle d_{0 \sigma} \mid a_{\mathbf{k}^{\prime} \sigma}^{+}\right\rangle\right\rangle_{\omega}+\sum_{\mathbf{q}} \Delta_{\mathbf{q} \sigma}\left\langle\left\langle a_{\mathbf{k}-\mathbf{q}, \sigma} \mid a_{\mathbf{k}^{\prime} \sigma}^{+}\right\rangle\right\rangle_{\omega} \\
& \left(\omega-t_{\mathbf{k}}\right)\left\langle\left\langle a_{\mathbf{k} \sigma} \mid d_{0 \sigma}^{+}\right\rangle\right\rangle_{\omega}=V_{\mathbf{k}}\left\langle\left\langle d_{0 \sigma} \mid d_{0 \sigma}^{+}\right\rangle\right\rangle_{\omega}+\sum_{\mathbf{q}} \Delta_{\mathbf{q} \sigma}\left\langle\left\langle a_{\mathbf{k}-\mathbf{q}, \sigma} \mid d_{0 \sigma}^{+}\right\rangle\right\rangle_{\omega} \\
& \left(\omega-E_{\sigma}\right)\left\langle\left\langle d_{0 \sigma} \mid d_{0 \sigma}^{+}\right\rangle\right\rangle_{\omega}=\frac{1}{2 \pi}+\sum_{\mathbf{k}} V_{\mathbf{k}}^{*}\left\langle\left\langle a_{\mathbf{k} \sigma} \mid d_{0 \sigma}^{+}\right\rangle\right\rangle_{\omega} \\
& \left(\omega-E_{\sigma}\right)\left\langle\left\langle d_{0 \sigma} \mid a_{\mathbf{k}^{\prime} \sigma}^{+}\right\rangle\right\rangle_{\omega}=\sum_{\mathbf{k}} V_{\mathbf{k}}^{*}\left\langle\left\langle a_{\mathbf{k} \sigma} \mid a_{\mathbf{k}^{\prime} \sigma}^{+}\right\rangle\right\rangle_{\omega} .
\end{aligned}
$$

From (14) the equations for Green functions $\left\langle\left\langle a_{\mathbf{k} \sigma} \mid a_{\mathbf{k}^{\prime} \sigma}^{+}\right\rangle\right\rangle_{\omega}$ are obtained

$$
\left(\omega-t_{\mathbf{k}}\right)\left\langle\left\langle a_{\mathbf{k} \sigma} \mid a_{\mathbf{k}^{\prime} \sigma}^{+}\right\rangle\right\rangle_{\omega}=\frac{1}{2 \pi} \delta_{\mathbf{k}, \mathbf{k}^{\prime}}+\sum_{\mathbf{q}} \Omega_{\mathbf{k}, \mathbf{q}}^{\sigma}\left\langle\left\langle a_{\mathbf{q} \sigma} \mid a_{\mathbf{k}^{\prime} \sigma}^{+}\right\rangle\right\rangle_{\omega} .
$$

Effective pseudopotential of electrons dispersion is

$$
\Omega_{\mathbf{k}, \mathbf{q}}^{\sigma}=\frac{V_{\mathbf{k}} V_{\mathbf{q}}^{*}}{\omega-E_{\sigma}}+\Delta_{\mathbf{k}-\mathbf{q}, \sigma} .
$$

The first term at (16) characterizes the intensity of resonant (non-elastic) dispersion, and the second term characterizes the elastic dispersion of band electrons on crystal heterogeneities.

From equation (15) Green function of zero approximation is obtained

$$
G_{0}^{\sigma}(\mathbf{k}, \omega)=\frac{1}{2 \pi} \frac{1}{\omega-t_{\mathbf{k}}-\Delta_{\sigma}-\frac{\left|V_{\mathbf{k}}\right|^{2}}{\omega-E_{\sigma}}}
$$

The parameter $\Delta_{\sigma}=\lim _{\mathbf{k} \rightarrow 0} \Delta_{\mathbf{k}, \sigma}=U_{\mathrm{s}}\left\langle n_{-\sigma}\right\rangle+\Delta E_{\mathrm{s}}\left(Z_{0}\right)$, where term

$$
\Delta E_{\mathrm{s}}\left(Z_{0}\right)=\frac{1}{N} \sum_{j=1}^{N}\left[\Phi_{\mathrm{a}}\left(\left|\mathbf{R}_{j}-\mathbf{R}_{0}\right|\right)+U\left(\left|\mathbf{R}_{j}-\mathbf{R}_{0}\right|\right)\left\langle n_{0}\right\rangle\right]
$$




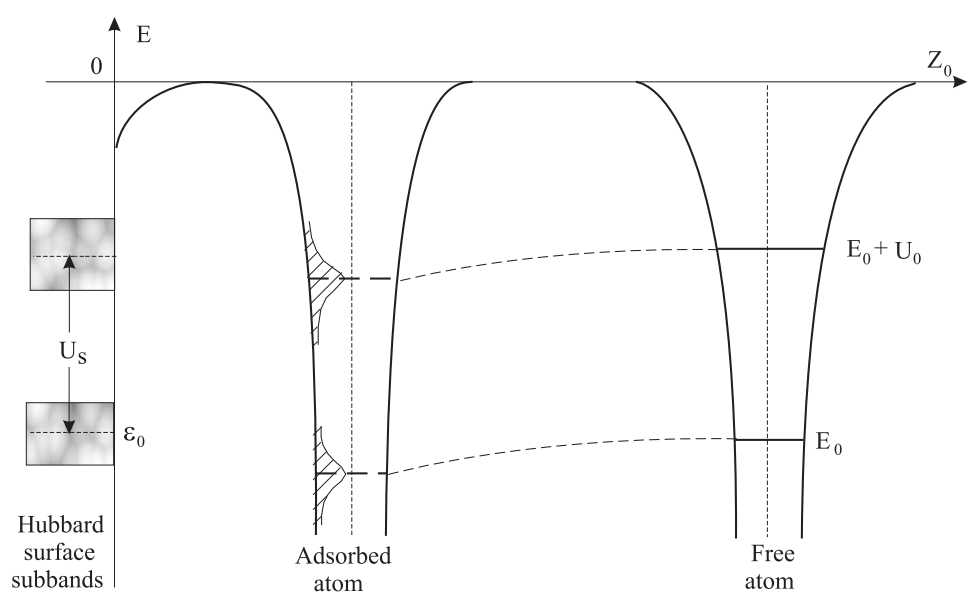

Figure 1. Energetic scheme of chemisorption.

describes homogeneous shift of surface band center $\mathcal{E}_{0}=N^{-1} \sum_{\mathbf{k}} t_{\mathbf{k}}$. Heterogeneous in surface plane wrap of conductivity band appears to more exact solution of integral equation (15). Electron dispersion law in this approximation is

$$
E_{1,2}^{\sigma}(\omega)=\frac{1}{2}\left[t_{\mathbf{k}}+\Delta_{\sigma}+E_{\sigma} \pm \sqrt{\left(t_{\mathbf{k}}+\Delta_{\sigma}-E_{\sigma}\right)^{2}+4\left|V_{\mathbf{k}}\right|^{2}}\right]
$$

For Green function $G_{00}^{\sigma}(\omega)=\left\langle\left\langle d_{0 \sigma} \mid d_{0 \sigma}^{+}\right\rangle\right\rangle_{\omega}$ from the system of equation (14) by dint of iteration we find an expression

$$
G_{00}^{\sigma}(\omega)=\frac{1}{2 \pi} \frac{1}{\omega-E_{\sigma}-\Sigma_{\sigma}(\omega)} .
$$

Self-energy part of Green functions is the series by power of pseudopotential dispersion

$$
\begin{aligned}
\Sigma_{\sigma}(\omega)= & \sum_{\mathbf{k}} \frac{\left|V_{\mathbf{k}}\right|^{2}}{\omega-t_{\mathbf{k}}}+\sum_{\mathbf{k}, \mathbf{q}(\mathbf{k} \neq \mathbf{q})} \frac{V_{\mathbf{k}}^{*} \Delta_{\mathbf{k}-\mathbf{q}, \sigma} V_{\mathbf{q}}}{\left(\omega-t_{\mathbf{k}}\right)\left(\omega-t_{\mathbf{q}}\right)} \\
& +\sum_{\mathbf{k}, \mathbf{q}, \mathbf{p}(\mathbf{k} \neq \mathbf{q} \neq \mathbf{p})} \frac{V_{\mathbf{k}}^{*} \Delta_{\mathbf{k}-\mathbf{q}, \sigma} \Delta_{\mathbf{q}-\mathbf{p}, \sigma} V_{\mathbf{q}}}{\left(\omega-t_{\mathbf{k}}\right)\left(\omega-t_{\mathbf{p}}\right)\left(\omega-t_{\mathbf{q}}\right)}+\ldots
\end{aligned}
$$

In the easiest approach, let us suggest that $\Delta_{\mathbf{k}, \sigma}=\Delta_{\sigma} \delta_{\mathbf{k}, 0}$, where $\delta_{\mathbf{k}, 0}$ is a Kronecker symbol. Such an approximation is equivalent in the theory of structural disordered system to the approximation of homogeneous effective media.

For this condition, the series (20) may be resumed and expression for Green function is derived

$$
G_{00}^{\sigma}(\omega)=\frac{1}{2 \pi}\left[\omega-E_{0}-U_{0} n_{a,-\sigma}-\Delta E_{\mathrm{a}}\left(Z_{0}\right)-\sum_{\mathbf{k}} \frac{\left|V_{\mathbf{k}}\right|^{2}}{\omega-t_{\mathbf{k}}-U_{\mathrm{s}} n_{s,-\sigma}-\Delta E_{\mathrm{s}}\left(Z_{0}\right)}\right]^{-1} .
$$


At this expression, the

$$
\Delta E_{\mathrm{a}}\left(Z_{0}\right)=\sum_{j=1}^{N}\left[\Phi_{\mathrm{s}}\left(\left|\mathbf{R}_{0}-\mathbf{R}_{j}\right|\right)+U\left(\left|\mathbf{R}_{0}-\mathbf{R}_{j}\right|\right)\left\langle n_{j}\right\rangle\right]
$$

is the adatom local level energy shift under the surface atoms total potential. At (22) $n_{a,-\sigma}=\left\langle\hat{n}_{0,-\sigma}\right\rangle$ is the averaged thermodynamic value of electrons number with spin projection $-\sigma$ that is localized on the adsorbed atom.

Doing at (22) the analytic continuation $\omega \rightarrow E+\mathrm{i} \varepsilon$ for Green function, we obtained

$$
G_{00}^{\sigma}(\omega)=\frac{1}{2 \pi}\left[E-E_{0}-U_{0} n_{a,-\sigma}-\Delta E_{\mathrm{a}}\left(Z_{0}\right)-\Lambda^{\prime}(E)+\mathrm{i} \Lambda^{\prime \prime}(E)\right]^{-1} .
$$

where

$$
\begin{aligned}
\Lambda^{\prime} & =\frac{1}{\pi} \mathrm{P} \int_{-\infty}^{+\infty} \frac{\Lambda^{\prime \prime}\left(E^{\prime}\right) \mathrm{d} E^{\prime}}{E-E^{\prime}-U_{\mathrm{s}} n_{s,-\sigma}-\Delta E_{\mathrm{s}}\left(Z_{0}\right)} \\
\Lambda^{\prime \prime} & =-\operatorname{Im} \sum_{\mathbf{k}} \frac{\left|V_{\mathbf{k}}\right|^{2}}{E-E_{\mathbf{k}}-U_{\mathrm{s}} n_{s,-\sigma}-\Delta E_{\mathrm{s}}\left(Z_{0}\right)+\mathrm{i} \epsilon} \\
& =\pi \sum_{\mathbf{k}}\left|V_{\mathbf{k}}\right|^{2} \delta\left(E-t_{\mathbf{k}}-U_{\mathrm{s}} n_{s,-\sigma}-\Delta E_{\mathrm{s}}\left(Z_{0}\right)\right)
\end{aligned}
$$

and $\mathrm{P}$ mean main sentence value of integral in Cauchy sense.

The adatom level position is received from transcendental equation

$$
E=E_{0}+U_{0} n_{a,-\sigma}+\Delta E_{\mathrm{a}}\left(Z_{0}\right)+\sum_{\mathbf{k}} \frac{\left|V_{\mathbf{k}}\right|^{2}}{E-t_{\mathbf{k}}-U_{\mathrm{s}} n_{s,-\sigma}-\Delta E_{\mathrm{s}}\left(Z_{0}\right)} .
$$

The width of the atomic energetic level is expressed by the imaginary part of Green function pole (23).

Adatom state is defined by charge $n=n_{a, \uparrow}+n_{a, \downarrow}$ and by the value of electron spin polarization $m=\left(n_{a, \uparrow}-n_{a, \downarrow}\right) / 2$. We can obtain these values from density of adatom states

$$
\rho_{\mathrm{a}}^{\sigma}(E)=\frac{1}{\pi} \operatorname{Im} G_{00}^{\sigma}(E)=\frac{1}{\pi} \frac{\Lambda^{\prime \prime}(E)}{\left(E-E_{0}-U_{0} n_{a,-\sigma}-\Delta E_{\mathrm{a}}\left(Z_{0}\right)-\Lambda^{\prime}(E)\right)^{2}+\left(\Lambda^{\prime \prime}(E)\right)^{2}},
$$

which have the form of Lorentz distribution. Then the averaged thermodynamic value of the number of electrons, localized at adatom is

$$
n_{a, \sigma}=\int_{-\infty}^{\mu} \rho_{\mathrm{a}}^{\sigma}(E) \mathrm{d} E
$$

where $\mu$ is the chemical potential of electron subsystem. 


\section{Conclusions}

In the present paper, the generalized model of atoms chemisorption with hydrogen-like electronic structure of valence envelope on crystalline surfaces based on microscopic Newns-Anderson model [6] is suggested. Our model differs from similar studies of other authors in the following aspects. Firstly, the superficial states are taken into account in the approach of tight-binding, that makes it possible to extend the model for chemisorption phenomenon description to dielectric and semiconductor surfaces with narrow surface bands. Secondly, the superficial spatialheterogeneousness of model parameters, the effects of local potential field of surface layer atoms, the Coulomb interaction at the "surface-adatom" system are taken into account. Finally, a self-consistent system of equations for calculating the charged and spin-polarized states of the adsorbed atom in Hartree-Fock approach is derived.

\section{References}

1. Gomer R. Some Problems of the Chemisorption Theory. - In: Surface Science. Cleveland, CRC Press, Inc., 1974.

2. Theory of Chemisorption. Edited by Smith J.R. Berlin-New York, Springer-Verlag, 1980.

3. Green M. Superficial Properties of Solid States. Moscow, Mir, 1980.

4. Zangwill E. Physics of Surface. Moscow, Mir, 1980.

5. Anderson P.W. // Phys. Rev., 1961, vol. 124. No. 1, p. 41-53.

6. Newns D.M. // Phys. Rev., 1969, vol. 178. No. 3, p. 1123-1135.

7. Schrieffer R., Gomer R. // Surf. Science, 1971, vol. 25, p. 315.

\section{Деякі аспекти наближення сильного зв'язку в теорії хемосорбції}

\section{Ю.Рудавський, Г.Понеділок, Ю.Петрів}

Державний університет “Львівська політехніка"

79013 Львів, вул. С.Бандери, 12

Отримано 26 серпня 2000 р.

Розглядається квантово-статистична задача хемосорбції атома 3 водневоподібною електронною структурою на кристалічних поверхнях напівпровідників та діелектриків. Поверхневі стани описуються наближенням сильного зв'язку. В наближенні Хартрі-Фока отримано самоузгоджену систему рівнянь для розрахунку величини зарядового та спін-поляризованого станів адсорбованого атома.

Ключові слова: адатом, хемосорбція, поверхня, енергія хемосорбції

PACS: $73.20 . H, 82.65 . Y$ 